\title{
Adolescentes en riesgo: factores asociados con el intento de suicidio en México*
}

\author{
Teenagers at Risk: Factors Associated to Suicide Attempts in Mexico \\ Adolescentes em risco: fatores associados à tentativa de suicídio no México
}

\author{
Marisol Luna Contreras ${ }^{a}$ \\ FLACSO, México \\ ORCID: http://orcid.org/0000-0002-7571-3449 \\ Claudio Alberto Dávila Cervantes \\ ORCID: http://orcid.org/0000-0002-7656-3606
}

DOI: https://doi.org/10.11144/Javeriana.rgsp17-34.arfa

\author{
Fecha de recepción: 24 Mayo 2017 \\ Fecha de aprobación: 14 Septiembre 2017
}

\section{Resumen:}

En México el suicidio ha aumentado desde hace más de cuarenta años. El objetivo fue analizar los factores asociados con el intento de suicidio en adolescentes mexicanos en tres momentos: los doce meses previos a la encuesta, antes de doce meses y en algún momento de la vida. Se ajustaron modelos de regresión logística binomial y se exploró el método del intento con un análisis de correspondencias múltiples. Las mujeres reportaron mayor prevalencia de intento de suicidio, y esta fue menor para los más jóvenes. Si sufrió una agresión o tenía rezago educativo aumentó la propensión a intentar suicidarse, al igual que en aquellos adolescentes que consumen alcohol o tabaco. La prevención del suicidio se debe enfocar en el intento, que es su principal factor de riesgo, y se debe dirigir hacia las mujeres más jóvenes, adolescentes con rezago educativo, que fumen o consuman alcohol o que sufrieron recientemente violencia.

Palabras clave: suicidio, México, intento de suicidio, adolescente, prevalencia, salud pública.

\begin{abstract}
:
In Mexico, suicides have increased for more than forty years ago. This article aims to analyze the factors associated to the suicide attempts by Mexican teenagers in three different time points: at twelve month before the survey; any time between the survey and the 12-month point; and any time in the teenager's life. Binomial logistic regression models were adjusted in order to explore the suicide attempt types under a multiple correspondence analysis. Women showed a higher suicide attempt prevalence, but it was lower among the younger ones. Having been assaulted or being in educational backwardness led to an increase in the suicidal intent; it was also an increase thereof among tobacco- or alcohol-consuming teenagers. Suicide prevention should be focused on the attempts, which is the main risk factor and prevention efforts should target the youngest women, those teenagers in educational backwardness who, either smoke or consume alcohol, or who underwent a violence event recently.
\end{abstract}

Keywords: suicide, Mexico, suicide attempt, teenager, prevalence, public health.

\section{Resumo:}

No México o suicídio vem se acrescentando por mais de quarenta anos. O objetivo foi analisar os fatores associados à tentativa de suicídio em adolescentes mexicanos em três momentos: os doze meses prévios ao inquérito, antes de doze meses e em algum momento da vida. Modelos de regressão logística binomial foram ajustados e explorou-se o método da tentativa com uma análise de correspondências múltiplas. As mulheres mostraram maior prevalência de tentativa de suicídio, e ela foi menor para os mais jovens. Quando tiver sofrido agressão ou atraso educativo, a propensão a tentar se suicidar aumentou, bem como em aqueles adolescentes que consomem álcool ou tabaco. A prevenção do suicídio deve se enfocar na tentativa, que é o principal fator de risco, e deve se direcionar para as mulheres mais jovens, adolescentes com atraso educativo, que fumem ou consumam álcool ou que sofreram recentemente violência.

Palavras-chave: suicídio, México, tentativa de suicídio, adolescente, prevalência, saúde pública. 


\section{Introducción}

El suicidio es uno de los principales problemas de salud pública a nivel mundial; representa cerca de la mitad de las muertes violentas para hombres y más de $70 \%$ para mujeres [1]. En el año 2000 se reportaron aproximadamente 815000 suicidios a nivel mundial, con una tasa de 14.5 suicidios por 100000 habitantes [2]; mientras que en 2012 se registraron 804000 casos (1.4\% del total de defunciones), con una tasa de 11.4 suicidios (15.0 entre hombres y 8.0 entre mujeres), ubicándose como la decimoquinta causa principal de muerte [1]. Se estima que el suicidio es, además, la segunda causa principal de muerte en el grupo de quince a veintinueve años a nivel mundial. Tres de cada cuatro suicidios ocurren en países de ingresos medios y bajos, por lo que dichos países enfrentan una enorme carga de mortalidad por esta causa [1].

En México, se ha dado un incremento sostenido e ininterrumpido de los casos de suicidio desde hace más de cuarenta años $[1,3,4,5]$. En 2015 se registraron 6425 muertes por suicidio en todo el país, con una tasa de mortalidad por suicidio de 5.31 suicidios por cada cien mil habitantes; mientras que en 1970 la tasa fue de 1.13 suicidios y en 1994 se incrementó a 2.89 [4]; se observa entonces, para este largo periodo, un crecimiento de la tasa por suicidios de $370 \%$. Dicho incremento de la mortalidad por suicidios se presentó principalmente en la población joven $[3,5,6]$. La razón hombre: mujer de suicidios en 2015 fue de 4.02, lo que implica que se presentaron cuatro defunciones por suicidio masculino por cada femenino. Actualmente, para la población de quince a veintinueve años el suicidio es la tercera causa de muerte en ambos sexos, solo por detrás de los homicidios y accidentes de vehículo automotor; mientras que, entre las edades de diez a diecinueve años, es la tercera causa de muerte para hombres y la primera para mujeres.

Estas tendencias actuales en México muestran que los jóvenes, y en particular los adolescentes, presentan un elevado riesgo de morir por suicidio y de realizar intentos de suicidio [5]. Es importante mencionar que la adolescencia se considera como un periodo de transición entre la niñez y la adultez temprana que se caracteriza por grandes cambios emocionales, sociales y físicos que ocurren durante este momento crucial del desarrollo [7], los cuales pueden facilitar el desarrollo de síntomas depresivos o conductas suicidas debido a la inhabilidad de enfrentar dichos cambios [8,9].

Un intento previo es el principal factor de riesgo del suicidio [1]. Se ha estimado que por cada suicidio consumado se producen de diez a cuarenta intentos $[3,10]$, los cuales constituyen una enorme carga social y económica debido a: la utilización de los servicios de salud para tratar las lesiones; tiene un elevado impacto psicológico y social; incrementa la probabilidad de intentos subsecuentes [11]; y, en ocasiones, se traduce en una discapacidad a largo plazo que es causa de un profundo sufrimiento psicológico [1]. La prevalencia de intento de suicidio alguna vez en la vida se ha visto que es mayor en la adolescencia y la juventud tanto en hombres como en mujeres [12].

$\mathrm{Al}$ igual que en el suicidio consumado, se ha observado un aumento de la conducta suicida en México, principalmente en adolescentes [13]. La conducta suicida es un continuo que abarca desde la ideación, la planificación, el intento y culmina con el suicidio consumado [14]. Es, por tanto, indispensable comprender la dinámica relacionada con el crecimiento del suicidio en México [15] a partir del estudio del intento de suicidio y sus factores de riesgo $[1,16,17]$, por lo que el principal objetivo de la presente investigación es analizar los principales factores asociados con el intento de suicidio en los adolescentes mexicanos en 2012.

\section{Método}

Se realizó un estudio observacional, de corte transversal y descriptivo con información de 21509 adolescentes de diez a diecinueve años. Se utilizó información de la Encuesta Nacional de Salud y Nutrición en México (Ensanut), la cual forma parte un conjunto de encuestas sobre salud realizadas desde 1987. La Ensanut 2012 tiene entre sus objetivos conocer las condiciones de salud y nutrición de la población mexicana, así como 
sus determinantes. Esta encuesta fue probabilística con representatividad nacional, estatal y para los estratos urbanos y rurales de México [18]. La recolección de la información del componente de salud se hizo mediante entrevista directa y se utilizaron los siguientes cuestionarios: utilización de servicios de salud; adultos de veinte o más años; menores de cero a nueve años; adolescentes de diez a diecinueve años; y hogar. Estos dos últimos cuestionarios fueron los utilizados para la realización de esta investigación.

En la Ensanut 2012 se indagó acerca del intento de suicidio y se planteó a los adolescentes entre diez y diecinueve años la pregunta: "Alguna vez en la vida, ¿has intentado o a propósito te has herido, cortado, intoxicado o hecho daño con el fin de quitarte la vida?", cuya respuesta distingue si dicho intento se realizó en los últimos doce meses o previamente a ese periodo, por lo cual se pudo estudiar el intento de suicidio en tres momentos del tiempo: 1) en los doce meses previos a la encuesta; 2) antes de los doce meses previos a la encuesta; 3) en algún momento de la vida, el cual toma en consideración los dos anteriores.

Las características sociodemográficas y de riesgo analizadas fueron: edad, escolaridad, estado conyugal, asistencia a la escuela, rezago educativo, tamaño de localidad de residencia del adolescente, haber tenido relaciones sexuales, el consumo de tabaco y alcohol, y haber vivido alguna situación de violencia o agresión por parte del novio, pareja, esposo o algún familiar o compañero escuela en los doce meses previos al levantamiento de la encuesta.

En el caso de la escolaridad, se optó por utilizar el indicador de rezago escolar, de manera que el adolescente estaba en situación de rezago escolar si para su edad no había alcanzado la escolaridad que le correspondía; para ello, se tomó como base que los adolescentes con diez años debían tener por lo menos tres años de escolaridad. Respecto al consumo de alcohol, se consideró que toma alcohol, si el adolescente había consumido alguna vez una bebida alcohólica; mientras que se consideró que el adolescente consumía tabaco si este refirió que había fumado por lo menos cien cigarros (cinco cajetillas) en toda su vida.

Primero se realizó un análisis descriptivo bivariado, el cual permitió calcular la prevalencia del intento de suicidio por sus principales factores sociales y demográficos asociados. Para esta parte del análisis se usaron tablas de contingencia y la prueba de independencia Ji-Cuadrada.

Se ajustaron dos modelos de regresión logística binomial (MRL), con el objetivo de analizar cuáles son las características de los adolescentes que incrementan la posibilidad de que hayan intentado quitarse la vida en los doce meses anteriores a la entrevista o en algún momento de la vida. En los MRL se incluyó la interacción entre las variables de edad y sexo. No se incluyó el modelo de regresión logística, en donde la variable por explicar fue el intento de suicidio en los últimos doce meses previos a la entrevista, ya que el ajuste de este no fue satisfactorio (el p-valor de la prueba $F$ fue de 0.0036).

Además, se exploró el método mediante el cual el adolescente intentó suicidarse, diferenciando por sexo y edad, pero solo se contó con la información si el evento tuvo lugar en los doce meses anteriores al levantamiento de la encuesta. Esto se llevó a cabo por medio del análisis de correspondencias múltiples (ACM), una técnica descriptiva de análisis multivariado que permite analizar la asociación entre los renglones y las columnas de una tabla de contingencia mediante una representación gráfica. De manera que este método ofrece la posibilidad de analizar, desde un punto de vista gráfico, las relaciones que existen entre un conjunto de variables categóricas [19].

Tanto para el análisis descriptivo como para el ajuste de los modelos de regresión logística se tomó en cuenta el efecto del diseño muestral de la encuesta. Esto se hizo con el paquete estadístico Stata versión 12.1. Además, se tuvo cuidado de que el coeficiente de variación de todas las estimaciones fuera inferior a $25 \%$, esto con la finalidad de que las estimaciones tuvieran un mayor grado de fiabilidad. 


\section{Resultados}

En 2012 la prevalencia de intento de suicidio de los adolescentes mexicanos de diez a diecinueve años alguna vez en la vida fue de $2.74 \%$, de la cual $1.45 \%$ se dio en los doce meses anteriores al levantamiento de la encuesta y el $1.29 \%$ tuvo lugar previamente a este periodo (tabla 1). La intensidad del intento de suicidio fue más frecuente entre las adolescentes, puesto que las prevalencias encontradas fueron 4.4 (en el último año), 6.9 (antes de los doce meses previos a la encuesta) y 5.4 (alguna vez en la vida) veces mayores que las observadas entre sus pares masculinos. La mayor prevalencia de intento de suicidio en los tres momentos analizados se observó para el grupo de edad de catorce a dieciséis años. La prevalencia de este grupo de edad solo fue estadísticamente diferente de la prevalencia del grupo de edad de diez a trece años si el intento de suicidio se dio antes de los doce meses previos al levantamiento de la encuesta (la prevalencia entre el grupo de catorce a dieciséis años es 4.8 veces mayor en comparación con el grupo de adolescentes más jóvenes) y alguna vez en la vida (la prevalencia del grupo de catorce a dieciséis años es 2.3 veces mayor en comparación con la del grupo de edad diez a trece años). 
TABLA 1

México. Diferenciales del intento de suicidio en adolescentes de diez a diecinueve años según características seleccionadas, 2012

\begin{tabular}{|c|c|c|c|c|c|c|}
\hline & \multicolumn{6}{|c|}{ Intento de suicidio } \\
\hline & \multicolumn{2}{|c|}{$\begin{array}{l}\text { Sí, en los últimos } 12 \\
\text { meses }\end{array}$} & \multicolumn{2}{|c|}{$\begin{array}{c}\text { Si, pero no en los últimos } \\
12 \text { meses }\end{array}$} & \multicolumn{2}{|c|}{ Alguna vez en la vida } \\
\hline & $\%$ & IC $95 \%$ & $\%$ & IC $95 \%$ & $\%$ & IC $95 \%$ \\
\hline Total & 1.5 & {$[1.2,1.7]$} & 1,3 & {$[1.0,1,6]$} & 2,7 & {$[2.4,3.1]$} \\
\hline \multicolumn{7}{|l|}{ Sexo*** } \\
\hline Hombre & 0.5 & {$[0.4,0.8]$} & 0.3 & {$[0.2,0.6]$} & 0.9 & {$[0.6,1.2]$} \\
\hline Mujer & 2.4 & {$[2.0,2.9]$} & 2.3 & {$[1.8,2.8]$} & 4.6 & {$[4.0,5.3]$} \\
\hline \multicolumn{7}{|l|}{ Edad**** } \\
\hline oct-13 & 1.2 & {$[0.9,1.6]$} & 0.4 & {$[0.3,0.6]$} & 1.6 & {$[1,2,2.1]$} \\
\hline $14-16$ & 1.7 & {$[1.3,2.1]$} & 1.9 & {$[1.4,2.6]$} & 3.6 & {$[2.9,4.3]$} \\
\hline $17-19$ & 1.6 & {$[1.1,2.3]$} & 1.8 & {$[1.3,2.5]$} & 3.4 & {$[2.8,4.4]$} \\
\hline \multicolumn{7}{|l|}{ Estado conyugal *** } \\
\hline Nunca unido & 1.4 & {$[1.2,1.7]$} & 1.1 & {$[0.9,1.4]$} & 2.5 & {$[0.6,1.2]$} \\
\hline Unido actualmente o alguna vez & 1.9 & {$[1.0,3.5]$} & 4.2 & {$[2.4,7.0]$} & 6.1 & {$[0.6,1.2]$} \\
\hline \multicolumn{7}{|l|}{ Rezago educativo** } \\
\hline No & 1.3 & {$[1.1,1.6]$} & 1.1 & {$[0.9,1.4]$} & 2.4 & {$[2.1,2.8]$} \\
\hline $\mathrm{Si}$ & 1.9 & {$[1.4,2.5]$} & 1,9 & {$[1.3,2.8]$} & 3.8 & {$[3.0,4.7]$} \\
\hline \multicolumn{7}{|l|}{ Asistencia actual a la escuela* } \\
\hline No & 1.6 & {$[1.2,1.6]$} & 1.9 & {$[1.3,2.8]$} & 3.6 & {$[2.8,4.6]$} \\
\hline $\mathrm{Si}$ & 1.4 & {$[1.1,1.7]$} & 1.1 & {$[0.9,1.4]$} & 2.5 & {$[2.1,2.9]$} \\
\hline \multicolumn{7}{|l|}{ Tamaño de la localidad* } \\
\hline Rural & 1.2 & {$[0.9,1.6]$} & 0.9 & {$[0.6,1.3]$} & 2.1 & {$[1.6,2.7]$} \\
\hline Urbano & 1.5 & {$[1.2,1.9]$} & 1.4 & {$[1.1,1.8]$} & 3.0 & {$[2.6,3.4]$} \\
\hline \multicolumn{7}{|l|}{ Ha tenido relaciones sexuales ${ }^{+} * * *$} \\
\hline No & 1.7 & {$[1.4,2.1]$} & 1.2 & {$[0.9,1.5]$} & 2.9 & {$[2.5,3.4]$} \\
\hline Si & 1.8 & {$[1.3,2.5]$} & 2.9 & {$[2.0,4.2]$} & 4.7 & {$[3.7,6.1]$} \\
\hline \multicolumn{7}{|c|}{ Consumo de por lo menos 5 cajetillas de cigarros*** } \\
\hline No & 1.2 & {$[1.0,1.5]$} & 1.1 & {$[0.9,1.4]$} & 2.4 & {$[2.1,2.7]$} \\
\hline $\mathrm{Si}$ & 3.4 & {$[2.5,4.8]$} & 2.8 & {$[1.8,4.3]$} & 6.3 & {$[4.8,8.1]$} \\
\hline \multicolumn{7}{|l|}{ Consumo de alcohol*** } \\
\hline No & 0.7 & {$[0.5,1.0]$} & 0.6 & {$[0.4,0.8]$} & 1.3 & {$[1.0,1.7]$} \\
\hline $\mathrm{Si}$ & 2.4 & {$[1.9,2.9]$} & 2.2 & {$[1.8,2.8]$} & 4.6 & {$[4.0,5.4]$} \\
\hline \multicolumn{7}{|c|}{ Violencia por novio/pareja/esposo, algún familiar o compañero de la escuela*** } \\
\hline No & 1.4 & {$[1.2,1.7]$} & & & & \\
\hline $\mathrm{Si}$ & 7.9 & {$[4.2,14.3]$} & & & & \\
\hline
\end{tabular}

Fuente: elaboración propia con base en la Ensanut-2012.

++ Solo se toma en cuenta a niños y adolescentes de entre doce y diecinueve años.

Significancia de la prueba de independencia Ji-Cuadrada ${ }^{*} \mathrm{p}<0.05,{ }^{* *} \mathrm{p}<0.01,{ }^{* * *} \mathrm{p}<0.001$.

La prevalencia de intento de suicidio en los doce meses anteriores al levantamiento de la información fue similar entre el grupo de adolescentes célibes y sus pares que estaban unidos o estuvieron alguna vez unidos. Esto no ocurrió si el intento de suicidio se dio en otro momento, donde la prevalencia observada es 3.7 veces mayor entre los adolescentes que ya habían contraído nupcias, en comparación con los solteros (4.2\% vs. $1.1 \%)$. La prevalencia de intento de suicidio alguna vez en la vida de los adolescentes alguna vez unidos fue de $6.1 \%$. 
Las prevalencias encontradas para el intento de suicido, sin importar el momento en que este ocurrió, no son estadísticamente diferentes entre los adolescentes que asistían a la escuela y entre quienes habían salido del sistema educativo. Para el rezago educativo, las diferencias entre los adolescentes que sí habían alcanzado la escolaridad para su edad y los que no, solo son significativas para el intento de suicidio alguna vez en la vida: de cada cien adolescentes con rezago educativo, cuatro han intentado suicidarse, valor que disminuye a menos de tres de cada cien entre los no rezagados académicamente. En los tres momentos analizados del intento de suicidio, las diferencias en las prevalencias entre los adolescentes que residen en una localidad rural o en una urbana resultaron no ser estadísticamente diferentes.

Respecto a los factores de riesgo, las mayores prevalencias de intento de suicidio, sin importar el momento en que este ocurrió, se encuentran entre los adolescentes que consumen tabaco, que han consumido alcohol o que vivieron algún episodio de violencia. Para estos últimos, de cada cien adolescentes que fueron violentados por el novio, pareja, esposo, familiar o compañero de la escuela, ocho intentaron quitarse la vida en el año previo al levantamiento de la encuesta. En el grupo de adolescentes que han consumido por lo menos cinco cajetillas de cigarros en su vida, las prevalencias son más del doble en comparación con sus pares que nunca han consumido tabaco o no han fumado esta cantidad de cigarros (2.8 veces en los últimos doce meses, 2.5 veces antes del año previo al levantamiento de la encuesta y 2.6 veces para alguna vez en la vida). La prevalencia de intento de suicidio entre los adolescentes que ya han tomado bebidas alcohólicas es tres veces mayor que la de aquellos que no han consumido alcohol: 3.4 si el intento de suicidio se dio en los últimos doce meses, 3.7 veces cuando ocurrió antes del año previo al levantamiento de la encuesta; y 3.6 veces para el intento de suicidio alguna vez en la vida.

Haber tenido relaciones sexuales no constituyó un factor que marcara diferencia en la prevalencia de intento de suicidio, si este ocurrió en el último año. Por el contrario, si este evento tuvo lugar anteriormente, la prevalencia fue más del doble (2.8\% vs. $1.1 \%)$ entre los adolescentes que ya tuvieron su primera relación sexual y los que no, mientras que la prevalencia de suicidio alguna de vez en la vida en adolescentes que han tenido relaciones sexuales fue de $4.7 \%$.

Otro resultado del análisis bivariado fue que la prueba de independencia Ji-Cuadrada fue significativa para todas las características analizadas, sin importar el momento en el cual ocurrió el intento de suicidio. Esto implica que hay dependencia estadística entre las características por analizar y el intento del suicidio. 


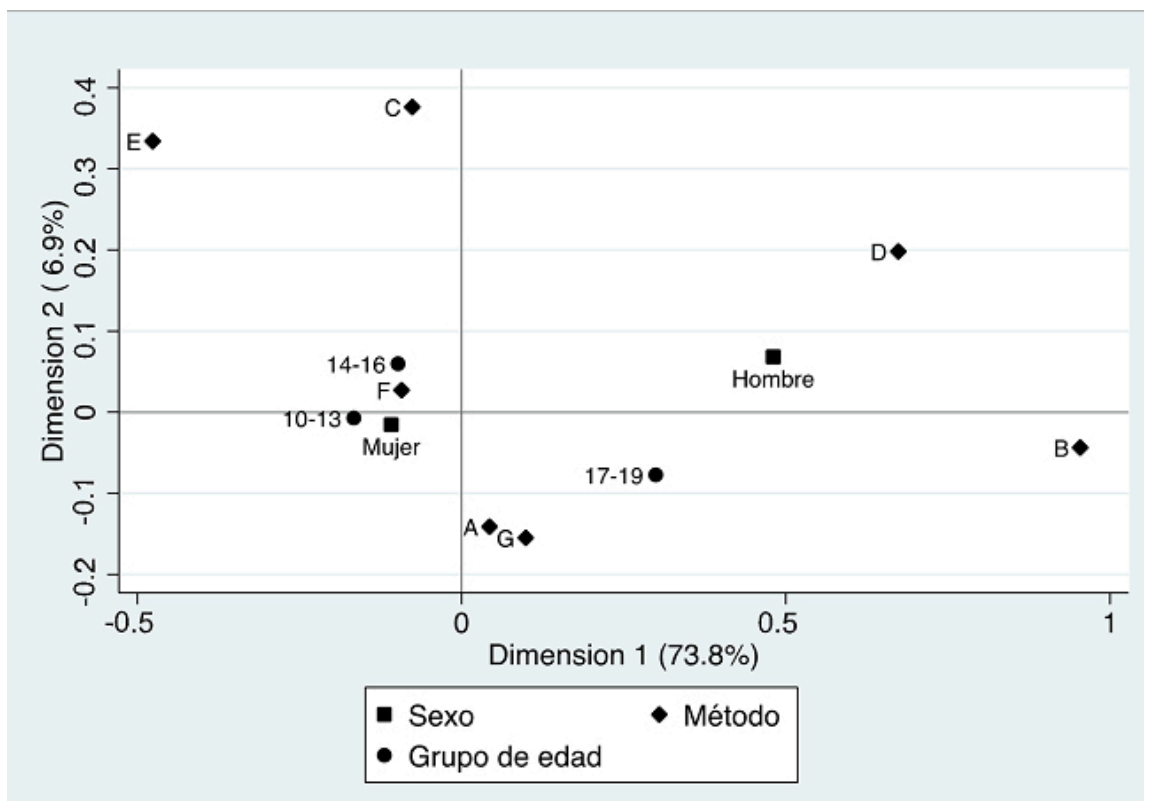

FIGURA 1

México. Método mediante el cual los adolescentes de diez a diecinueve años intentaron suicidarse, 2012

$\mathrm{A}=$ envenenamiento con medicamentos; $\mathrm{B}=$ envenenamiento con narcóticos o alcohol; $\mathrm{C}=$ envenenamiento por inhalación de hidrocarburos, fumigantes, insecticidas o con productos químicos, ácidos, corrosivos; $\mathrm{D}=$ ahorcamiento; $\mathrm{E}=$ quemaduras $; \mathrm{F}=$ objetos punzocortantes; $\mathrm{G}=$ arrojarse al vacío o de un vehículo en movimiento. Fuente: elaboración propia con base en la Ensanut-2012.

Los resultados del ACM sobre el método mediante el cual los adolescentes intentaron quitarse la vida en los doce meses previos al levantamiento de la encuesta, mostraron que con la primera dimensión se agrupa la mayor parte de la varianza explicada ( $73.8 \%$ con la dimensión 1 y $6.9 \%$ con la dimensión 2$)$, lo cual en este tipo técnicas es considerado satisfactorio. Se puede apreciar cómo la distancia entre la categoría de hombres y mujeres es amplia, así como entre los grupos de edad de 10-13 y 14-16 años con respecto al grupo de 17-19 años (figura 1). Esto implica que el comportamiento en la elección del método utilizado con el cual intentaron suicidarse es diferente en función del sexo y la edad, o lo que es lo mismo, hombres y mujeres eligen métodos diferentes para suicidarse y además de que lo hacen a edades diferentes. El comportamiento de las mujeres adolescentes destaca principalmente por dos razones: 1) el intento de suicidio se da a edades más tempranas (10-13 y 14-16 años); 2) es más diverso el método mediante el cual intentaron suicidarse, siendo los objetos punzocortantes, el envenenamiento con medicamentos y el arrojarse a algún vehículo en movimiento, los métodos a los cuales recurren más. Por su parte, los mecanismos más utilizados por los adolescentes son el ahorcamiento y el envenenamiento con narcóticos o alcohol.

El modelo de regresión logística mostró que el rezago educativo es un factor que incrementa el intento de suicido entre los adolescentes mexicanos de diez a diecinueve años. De manera que si los adolescentes no tienen los años de escolaridad acordes a su edad, se incrementó la posibilidad de realizar un intento de suicidio en últimos doce meses en 1.5 veces y 1.6 veces para alguna vez en la vida, en comparación con los adolescentes sin rezago educativo y controlando por el resto de las variables (tabla 2 ). 
TABLA 2

México. Modelo regresión logística según momento en el cual se dio el intento de suicidio en adolescentes de diez a diecinueve años, 2012

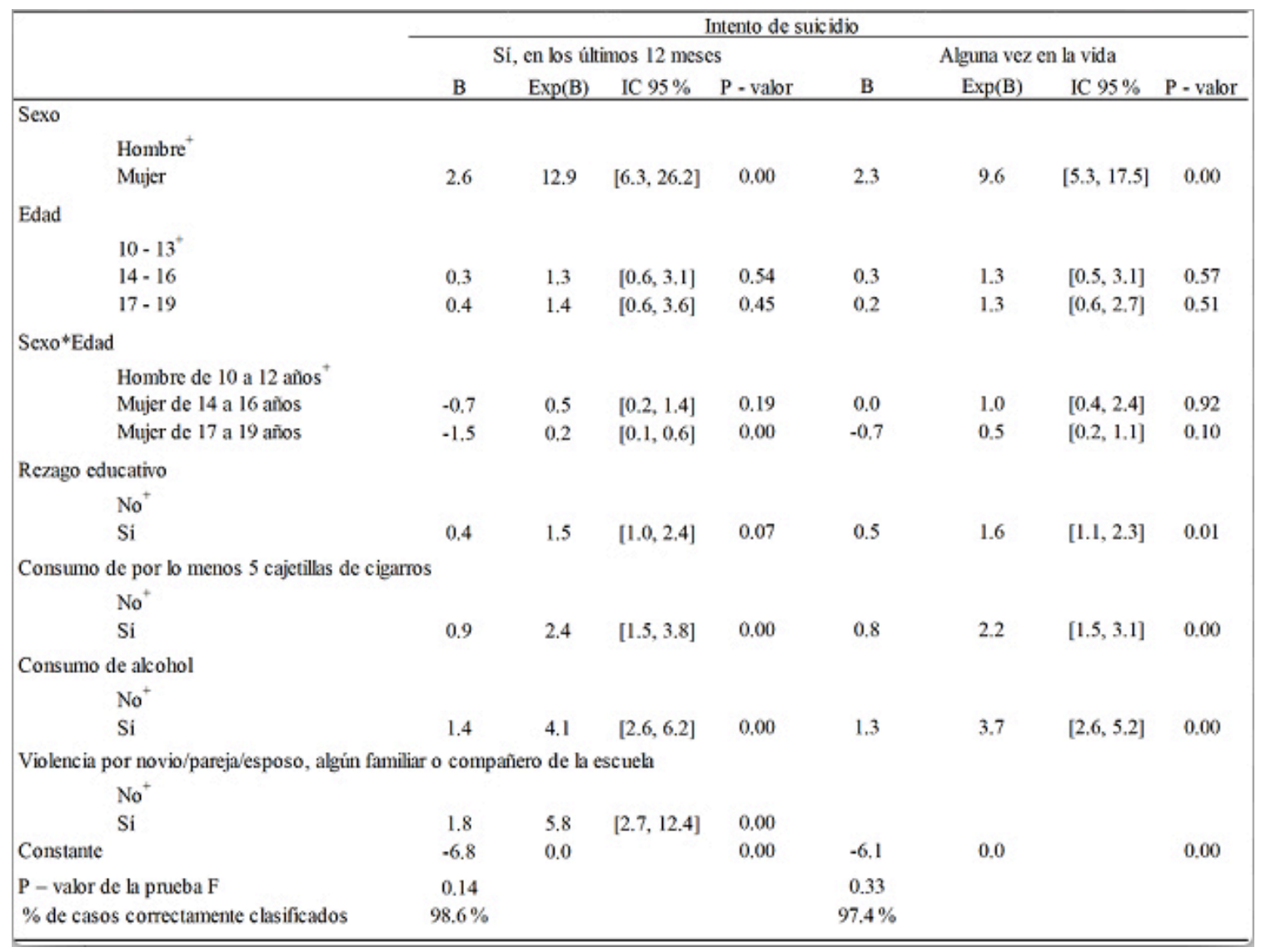

Fuente: elaboración propia con base en la Ensanut-2012 + Categoría de refe rencia

Controlado por las características sociodemográficas (edad, sexo y rezago educativo), se encontró que el consumo de alcohol y el consumo de tabaco resultaron ser factores que incrementan la propensión del intento de suicido en los dos momentos del tiempo analizados: si el adolescente ha consumido por lo menos cinco cajetillas de cigarros en su vida, tuvo 2.4 veces más posibilidades de intentar quitarse la vida en los últimos doce meses, en comparación con sus pares que nunca han fumado o que lo han hecho menos de esa cantidad; se encontró un valor similar para el intento de suicidio alguna vez en la vida (2.2 veces). Por otro lado, el consumo de alcohol aumentó la posibilidad de intento de suicidio entre los adolescentes en 4.1 veces cuando este ocurrió en el periodo más reciente analizado y 3.7 veces alguna vez en la vida.

Si el adolescente sufrió algún evento de violencia o agresión por parte del novio, pareja, esposo, algún familiar o compañero de la escuela en los doce meses previos al levantamiento de la información, incrementó en 5.8 veces la posibilidad de intento de suicidio en el mismo periodo, cuando se compara con los que no vivieron situaciones de violencia.

Como se observó en el ACM, hombres y mujeres tienen un comportamiento diferencial por edad ante el intento de suicidio. Por ello, en los MRL se incluyó una interacción entre el sexo y la edad. Los resultados en los dos modelos apuntan a que la edad a la cual los adolescentes intentaron suicidarse varía según el sexo de la persona. Aunque solo fue estadísticamente significativo para el grupo de mujeres de diecisiete a 
diecinueve años, se observa que conforme aumenta la edad, la posibilidad de que las mujeres adolescentes intenten quitarse la vida disminuye.

Una interpretación más detallada y clara de estos resultados se pudo observar con las probabilidades y efectos marginales obtenidos para el intento de suicidio por sexo y grupo de edad; para su obtención se mantuvo en su valor medio el resto las variables. Los resultados de la tabla 3 apuntan a que para cualquier grupo de edad es más probable que una mujer adolescente intente suicidarse que un hombre adolescente, sin importar el momento en el cual ocurrió el evento. Entre las mujeres existe una relación inversa entre la edad y la probabilidad de intento de suicidio cuando se consideran solo los últimos doce meses, con una mayor probabilidad en el grupo de edad de diez a trece años (3.1\%). En cambio, el comportamiento de intento de suicidio alguna vez en la vida entre las adolescentes en función de la edad no ocurre de forma lineal, puesto que la probabilidad aumenta $0.9 \%$ entre el grupo de edad catorce a dieciséis y diez a trece años, y disminuye $2.3 \%$ entre los grupos etarios de diecisiete a diecinueve años y de catorce a dieciséis años. Las probabilidades observadas en el grupo de los hombres se mantienen casi constantes entre los grupos etarios, en los dos momentos del tiempo.

TABLA 3

México. Probabilidades y efectos marginales del intento de suicidio según momento en el cual se dio el evento en adolescentes de diez a diecinueve años por sexo y grupos de edad, 2012

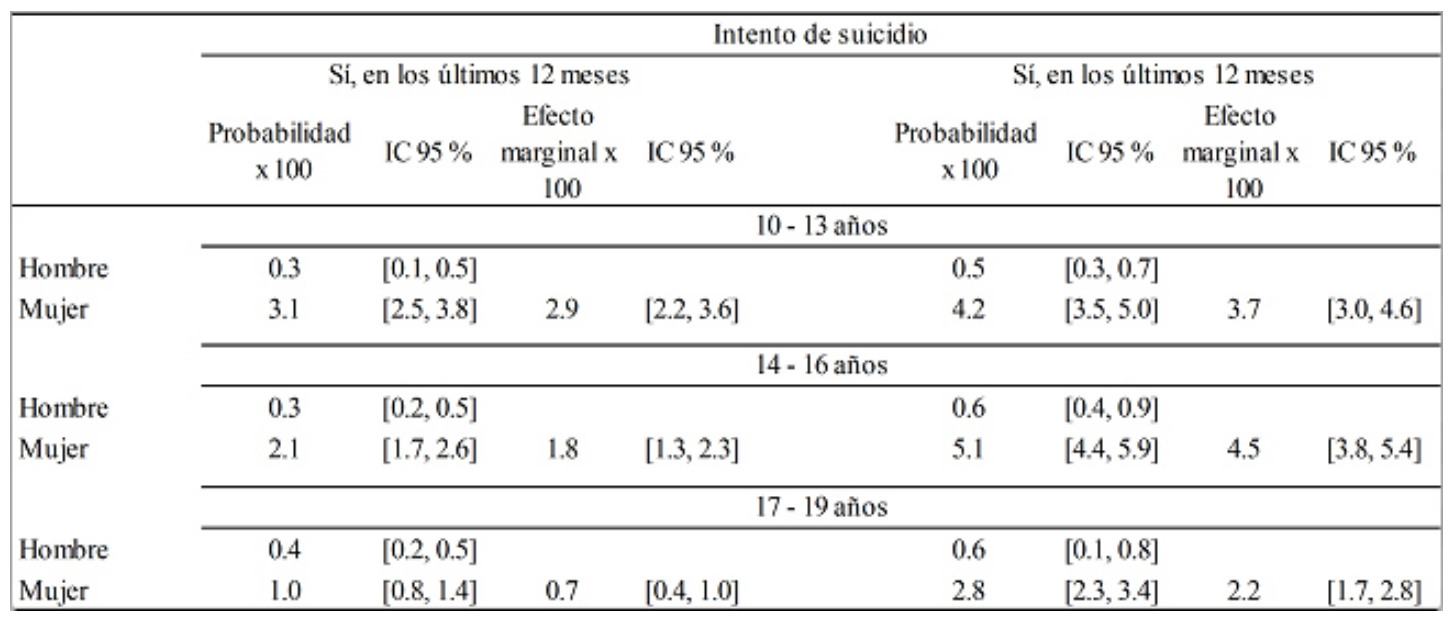

Fuente: elaboración propia con base en la Ensanut-2012.

En lo que respecta a la diferencia de las probabilidades de intento de suicidio entre mujeres y hombres, se observó que en los últimos doce meses esta se va acortando conforme aumenta la edad: en el grupo de edad diez a trece años la diferencia es de $2.9 \%$, disminuye a $1.8 \%$ en el grupo de edad de catorce a dieciséis años y a $0.7 \%$ en el grupo de mayor edad. En el intento de suicidio que se dio alguna vez en la vida no se encontró un patrón definido.

\section{Discusión}

El suicidio en la adolescencia es actualmente uno de los principales problemas de salud pública; lo es por la mortalidad prematura, que conlleva una enorme carga económica, social y psicológica para las personas, las familias y la sociedad en general [20,21]; y por sus altos costos sociales y económicos, ya que los intentos de suicidio tienen secuelas psicológicas y físicas graves y, en ocasiones, permanentes en el afectado. Durkheim consideró que el suicidio se puede considerar un reflejo de las deficientes condiciones de vida de una 
población, ya que la conducta suicida se presenta en sociedades con alta desintegración y falta de cohesión social $[22,23]$.

La prevalencia de intento de suicidio, tanto en los doce últimos meses como en alguna vez en la vida observada en esta investigación, fue mayor que aquella reportada en la Ensanut de 2006 para los adolescentes de entre diez y diecinueve años [12]. Sin embargo, fue menor a otras obtenidas en estudios realizados en población adolescente a nivel nacional en 2002 [5]; y en la Zona Metropolitana de la Ciudad de México en 2005 [2] y 2006 [5]. De igual forma, la prevalencia observada de intento de suicidio alguna vez en la vida se encuentra en el rango reportado en otras investigaciones, realizadas en otros contextos, en las cuales dicho valor varió entre un $0.72 \%$ y $5.93 \%$ [25,26]. En cambio, la prevalencia de suicidio en los últimos doce meses en adolescentes fue mayor que aquella reportada en México en 2008 en otros estudios [5,27].

El intento de suicidio es un fenómeno multicausal. En esta investigación, al igual que en otras, se detectó que las mujeres reportan una mayor prevalencia de intento de suicidio [5,24]; cuestión que se confirma controlando por las otras variables utilizadas en los modelos, observándose que para cualquier grupo de edad es más probable que una mujer adolescente intente suicidarse. Esta característica del fenómeno se ha explicado aludiendo a que, entre los adolescentes, las mujeres tienen una mayor predisposición a la depresión, lo que las pone en un mayor riesgo de presentar una conducta suicida [28,29]. De igual forma, se observó que la edad a la cual los adolescentes intentaron suicidarse es diferencial por sexo. En el caso de los hombres, la propensión de realizar un intento de suicidio se mantiene casi constante entre los grupos etarios; mientras que en mujeres conforme aumenta la edad, la posibilidad de que intenten quitarse la vida disminuye. Dichos resultados son distintos a los presentados en otras investigaciones, en las cuales el mayor riesgo de conducta suicida se presenta en la adolescencia tardía [21], lo que se ha relacionado con una mayor exposición a factores de riesgo, como trastornos de ánimo, que los adolescentes más jóvenes [30].

Se indagó también si aquellos adolescentes que declararon haber sufrido una agresión o violencia en los últimos doce meses tuvieron una mayor prevalencia de intento de suicidio, y se observó que en aquellos que sufrieron una agresión o violencia se incrementó la propensión a realizar un intento de suicidio. Esto se asemeja a los resultados de otros estudios, en los cuales reportan que los acontecimientos vitales estresantes o eventos traumáticos (como acoso [bullying], asalto, abuso o agresión sexual), los cuales son relativamente comunes en adultos y adolescentes en México [31], se asocian con el intento suicida [21,28,29,32,33,34]. Sin embargo, no existe todavía un consenso sobre la relación entre los eventos traumáticos y el intento de suicidio [35]. Se ha planteado que dicha asociación no es directa, sino que los acontecimientos traumáticos incrementan la probabilidad de que las personas desarrollen desórdenes psiquiátricos [36], los cuales a su vez aumentan la prevalencia de conducta suicida [37,38,39]; sin embargo, también se ha reportado un mayor riesgo de conducta suicida en adolescentes que sufrieron algún evento traumático, controlando por desórdenes psiquiátricos [35].

$\mathrm{Al}$ igual que en otras investigaciones, se observó que el rezago educativo se relaciona con el intento de suicidio en adolescentes mexicanos, incrementando la propensión a realizar un intento si se presenta una situación de rezago. Si bien se ha estudiado el nivel de escolaridad como un factor de riesgo del intento de suicidio en adolescentes y adultos jóvenes, la evidencia sobre dicha relación es todavía inconsistente [40]. Por un lado, se ha establecido que existe una relación inversa entre la escolaridad y la conducta suicida en general [41] y con el intento de suicidio en particular [38]. Pero, se ha argumentado también que los bajos niveles de escolaridad se relacionan con el suicidio solamente de manera indirecta, ya que reflejan una serie de factores desventajosos del contexto del adolescente y adulto joven, como son bajo estatus socioeconómico, bajo ingreso personal o familiar y la presencia de desórdenes psiquiátricos (incluidos desórdenes relacionados con abusos de sustancias) [40]).

Otro factor de riesgo importante del intento de suicidio es el consumo de alcohol y tabaco. En este estudio se observa que aquellos adolescentes que reportaron consumir alcohol o tabaco presentaron una mayor prevalencia de intento de suicidio, independientemente de cuándo se presentó el intento; dicha relación 
continuó después de controlar por el resto de las variables del modelo, aspecto en el cual los resultados aquí presentados muestran consistencia con otras investigaciones [33,42,43]. Esta asociación se ha encontrado incluso después de controlar por la presencia de desórdenes psiquiátricos [44]. La relación entre el consumo de alcohol y tabaco con el riesgo de desarrollar una conducta suicida se puede basar en que los adolescentes perciben estos comportamientos como una posible solución a eventos estresantes en la vida diaria [45], o bien porque el consumo de alcohol y tabaco es posible que sea un signo de la presencia de desorden de abuso de sustancias en los adolescentes.

Es importante mencionar que el suicidio se puede prevenir. Esto es relevante dado su incremento constante en México desde hace más de cuarenta años, el cual se debe considerar un llamado atención a la necesidad de planear y aplicar medidas preventivas [27]. Dichos esfuerzos preventivos deben poner especial énfasis en el intento de suicidio [46] que, como se mencionó previamente, es el principal factor de riesgo del suicidio consumado [47]. Por ello, es de gran importancia identificar y analizar los elementos o principales factores asociados que llevan al intento de suicidio [5].

\section{Limitaciones}

Los resultados de este estudio se deben interpretar a la luz de ciertas limitaciones importantes. En primer lugar, el análisis se realizó con base en autorreportes retrospectivos, por lo que es posible que, dada la característica estigmatizante de la conducta suicida, la prevalencia del intento de suicidio esté subestimada [27,48], y que algunos factores de riesgo pudieran variar después del intento de suicidio de los adolescentes. En segundo lugar, aunque se examinó un amplio número de factores de riesgo potenciales del intento de suicidio, algunos no pudieron ser incluidos en este estudio, entre los que destacan: la ideación suicida, la cual es uno de los principales precursores del intento de suicidio [20]; intentos previos de suicidio, ya que se ha reportado que un elevado número de los adolescentes que llevan a cabo un intento de suicidio serio han cometido al menos un intento previo [49]; tampoco se pudo analizar la prevalencia de intento de suicidio en presencia de algún trastorno mental, como la depresión [50] o desórdenes de personalidad [51], por falta de dicha información en la Encuesta Nacional de Salud y Nutrición 2012. En tercer lugar, se realizó un estudio de corte transversal, con lo cual no se pudo considerar la temporalidad y el orden con los cuales ocurrieron las agresiones o la violencia sufrida, intentos previos de suicidio o el inicio de la ideación suicida [35] y, por tanto, no es posible atribuir asociaciones causales entre los factores de riesgo y el intento de suicidio [20]. En cuarto lugar, si bien el recuerdo retrospectivo de adversidades, tales como la violación y el abuso sexual, se redujo a los últimos doce meses, su reporte puede haber sido influenciado por un intento suicida reciente [50].

\section{Consideraciones finales}

Dado el sostenido incremento de la mortalidad por suicidio en México, es indispensable la implementación de acciones para la detección, el tratamiento y la prevención de la conducta suicida para reducir su prevalencia en edades tempranas como la adolescencia [5]. Los resultados de esta investigación apuntan a la necesidad de dirigir los esfuerzos de prevención de comportamientos suicidas, en particular el intento de suicidio, en adolescentes con un enfoque en las mujeres entre los diez y dieciséis años, y los adolescentes con rezago educativo, que fumen o consuman alcohol, y, especialmente, si han sufrido algún episodio reciente de violencia. La importancia de identificar aquellos elementos que incrementan el riesgo de desencadenar un intento de suicidio radica en la posibilidad de implementar acciones necesarias y eficaces para prevenirlo [52]. 


\section{Referencias bibliográficas}

1. Organización Mundial de la Salud (OMS). Prevención del suicidio un imperativo global. Washington D.C.: OMS; 2014.

2. Organización Mundial de la Salud (OMS). Informe mundial sobre la violencia y la salud. Ginebra: OMS; 2002.

3. Hernández-Bringas HH, Flores-Arenales R. El suicidio en México. Papeles Poblac. 2011; 17(68):69-101.

4. Borges G, Rosovsky H, Gómez C, Gutiérrez R. Epidemiología del suicidio en México de 1970 a 1994. Salud PublicaMex. 1996; 38(3):197-206.

5. Borges G, Orozco R, Benjet C, Medina-Mora ME. Suicidio y conductas suicidas en México: retrospectiva y situación actual. SaludPublica Mex. 2010; 52(4):292-304.

6. Bridge JA, Goldstein TR, Brent DA. Adolescent suicide and suicidal behavior. J Child Psychol Psychiatry. 2006; 47(3-4):372-94.

7. Braush AM, Muehlenkamp JJ. Body image and suicidal ideation in adolescents. Body Image. 2007; 4(2):207-12.

8. Aneshensel CS, Huba GJ. An integrative causal model of the antecedents and consequences of depression over one year. Research in Community \& Mental Health.1984; 4:35-72.

9. Beck AT. Depression causes and treatment. Philadelphia: University of Pennsylvania Press; 1972.

10. Spicer RS, Miller TR. Suicide acts in 8 states: incidence and case fatality rates by demographics and method. Am J Public Health. 2000; 90(12):1885-91.

11. Neeleman J, de Graaf R, Vollebergh W. The suicidal process; prospective comparison between earlyand later stages. J AffectDisord. 2004; 82(1):43-52.

12. Villalobos-Hernández AL, Guerrero-López CM, Hernández-Serrato MI, Palma-Coca O, Rojas-Martínez R, OlaizFernández G. Intento de suicidio en adolescentes mexicanos según la Encuesta Nacional de Salud y Nutrición, 2006 [internet]. 9th World Conference on Injury Prevention and Safety Promotion; 15 marzo -18 marzo 2008; Mérida, México [acceso: 24 de abril de 2017]. Disponible en: http://www.conac.actuarios.org.mx/Documento s/Publicaciones/ActuariosTrabajando/AT-2008Num02.pdf\#page $=8$

13. González-Forteza C, Berenzon-Gorn S, Tello-Granados AM, Facio-Flores D, Medina-Mora Icaza ME. Ideación suicida y características asociadas en mujeres adolescentes. Salud Publica Mex. 1998; 40(5):430-7.

14. Salvo L, Melipillán R. Predictores de suicidalidad en adolescentes. Ver Chil Neuro-psiquiat. 2008; 46(2):115-23.

15. Borges G, Benjet C, Orozco R, Medina-Mora ME. The growth of suicide ideation, plan and attempt among young adults in the Mexico City metropolitan area. Epidemiology and Psychiatric Sciences. 2016; (1):1-9.

16. Aseltine R, Demartino R. An outcome evaluation of the suicide prevention program. Am J Public Health. 2004; 94: 446-51.

17. Mann J, Apter A, Bertolete J, Beautrais A, Currier D, Haas A, et al. Suicide prevention strategies a systematic review. JAMA. 2005; 294(816):2064-74.

18. Romero-Martínez M, Shamah-Levy T, Franco-Núñez A, Villalpando S, Cuevas-Nasu L, Gutiérrez JP, RiveraDommarco JA. Encuesta Nacional de Salud y Nutrición 2012: diseño y cobertura. Salud PublicaMex. 2013; 55(supl 2):S332-S340.

19. Greenacre M. Correspondence analysis in practice. Boca Raton: Chapman \& Hall /CRC Interdisciplinary statistics series; 2007.

20. Pérez-Amezcua B, Rivera-Rivera L, Atienzo EE, De Castro F, Leyva-López A, Chávez-Ayala R. Prevalencia y factores asociados a la ideación e intento suicida en adolescentes de educación media superior de la República Mexicana. Salud Publica Mex. 2010; 52(4):324-33.

21. Vargas HB, Saavedra JE. Factores asociados con la conducta suicida en adolescentes. Rev Neuropsiquiatr. 2012; 75(1):19-28.

22. Borges G, Orozco R, Medina-Mora ME. Índice de riesgo para el intento suicida en México. Salud Pública Mex. 2012; 54: 595-606.

23. Durkheim E. El suicidio, estudio de sociología. Madrid: Akal; 2003. 
24. Borges G, Benjet C, Medina-Mora ME, Orozco R, Nock MK. Suicide ideation, plan and attempt in the Mexican Adolescent Mental Health Survey. Journal of the American Academy of Child and Adolescent Psychiatry. 2008; $47(1): 41-52$.

25. Weissman MM, Bland R, Canino GJ, Greenwalk S, Hwu HG, Joyce PR, et al. Prevalence of suicide ideation and suicide attempts in nine countries. Psychol Med. 1999; 29(1):9-17.

26. Nock MK, Borges G, Bromet EJ, Alonso J, Angermeyer M, Beautrais A, et al. Cross-national prevalence and risk factors for suicidal ideation, plans and attempts. Br J Psychiatry. 2008; 192(2):98-105.

27. Borges G, Medina-Mora ME, Orozco R, Oueda C, Villatoro J, Fleiz C. Distribution and socio-demographic determinants of the suicidal behavior in Mexico. Salud Ment. 2009; 32:413-25.

28. Waldvogel JL, Rueter M, Oberg CN. Adolescent suicide: risk factors and prevention strategies. Current Problems in Pediatric and Adolescent Health Care. 2008; 38(4):110-25.

29. Langhinrichsen-Rohling J, Friend J, Powell A. Adoles-cent suicide, gender, and culture: a rate and risk factor analysis. Aggression and Violent Behavior. 2009; 14(5):402-14.

30. Cañón-Buitrago SC. Factores de riesgo asociados a conductas suicidas en niños y adolescentes. Archivos de Medicina. 2011; 11(1):62-67.

31. Orozco R, Borges G, Benjet C, Medina-Mora ME, López-Carrillo L. Traumatic life events and posttraumatic stress disorder among Mexican adolescents: results from a survey. Salud Publica Mex. 2008; 50(Suppl 1):S29-37.

32. Delfabbro PH, Winefield HR, Winefield AH. Life-time and current suicide-ideation in Australian secondary school students: socio-demographic, health and psychological predictors. Journal of Affective Disorders. 2013; 151(2):514-24.

33. Park S. Gender-specific factors of suicide ideation amongadolescents in the republic of Korea: a nationally representativepopulation-based study. Archives of Psychiatric Nursing. 2013; 27(5):253-9.

34. Steele MM, Doey T. Suicidal behaviour in children and adolescents part I: etiology and risk factors. Can J Psychiatry. 2007; 52(S1):21-33S.

35. Borges G, Benjet C, Medina-Mora ME, Orozco R, Molnar RE, Nock MK. Traumatic events and suicide- related outcomes among Mexico City adolescents. Journal of Child Psychology and Psychiatry. 2008; 49(6):654-66.

36. Kaplow JB, Dodge KA, Amaya-Jackson L, Saxe GN. Pathways to PTSD, part II: Sexually abused children. Am J Psychiatry. 2005; 162(7):1305-10.

37. Borges G, Angst J, Nock MK, Ruscio AM, Walters EE, Kessler RC. A risk index for 12-month suicide attempts in the National Comorbidity Survey Replication (NCS-R). Psychological Medicine. 2006; 36:1747-57.

38. Kessler RC, Borges G, Walters EE. Prevalence of and risk factors for lifetime suicide attempts in the National Comorbidity Survey.Arch Gen Psychiatry. 1999; 56(7):617-26.

39. Nock MK, Kessler RC. Prevalence of and risk factors for suicide attempts versus suicide gestures: Analysis of the National Comorbidity Survey. Journal of Abnormal Psychology. 2006; 115(3):616-23.

40. Beautrais AL, Joyce PR, Mulder RT. Youth suicide attempts: a social and demographic profile. Australian and New Zealand Journal of Psychiatry. 1998; 32:349-57.

41. Borges G, Nock MK, Medina-Mora ME, Benjet C, Lara C, Chiu WT, et al. The epidemiology of suicide-related outcomes in Mexico. Suicide and Life-Threatening Behavior. 2007; 37(6):627-40.

42. Hallfors D, Waller MW, Ford CA, Halpern CT, Brodish PH, Iritani B. Adolescent depression and suicide risk: Association with sex and drug behavior. Am J Prev Med. 2004; 27(3):224-31.

43. Wang J, Deng X-J, Wang J-J, Wang X-W, Xu L. Substance use, sexual behaviours, and suicidal ideation and attempts among adolescents: findings from the 2004 Guangzhou Youth Risk Behaviour Survey. Public Health. 2009; 123(2):116-21.

44. Miller M, Borges G, Orozco R, Mukamal K, Rimm EB, Benjet C, et al. Exposure to alcohol, drugs and tobacco and the risk of subsequent suicidality: Findings from the Mexican Adolescent Mental Health Survey. Drug and Alcohol Dependence. 2011; 113(2-3):110-7. 
45. Souza LD, Ores L, Oliveira GT, Sica AL, Azevedo R, Tavares R, et al. Ideação suicida na adolescência: prevalência e fatores associados. Jornal Brasileiro de Psiquiatria. 2010; 59(4):286-92.

46. Mann JJ, Apter A, Bertolote J, Beautrais A, Currier D, Haas A, et al. Suicide prevention strategies: a systematic review. JAMA. 2005; 294(16):2064-74.

47. Neeleman J, de Graaf R, Vollebergh W. The suicidal process; prospective comparison between early and later stages. J Affect Disord. 2004; 82(1):43-52.

48. Asarnow JR, Baraff LJ, Berk M, Grob C, Devich-Navarro M, Suddath R, et al. Effects of an emergency department mental health intervention for linking pediatric suicidal patients to follow-up mental health treatment: A randomized controlled trial. Psychiatric Services. 2011; 62(11):1303-9.

49. Mullen D, Hendren R. El niño o adolescente suicida. En: Parmelee D, David R editores. Psiquiatría del niño y el adolescente. Madrid: HarcourtBrace; 1998. p. 229-40.

50. Borges G, Orozco R, Medina-Mora ME. Índice de riesgo para el intento suicida en México. Salud Pública Mex. 2012; 54(6):595-606.

51. Palacio C, García J, Diago J, Zapata C, Lopez G, Ortiz J, et al. Identification of Suicide Risk Factors in Medellín, Colombia: A Case-Control Study of Psychological Autopsy in a Developing Country. Archives of Suicide Research. 2007; 11(3):297-308.

52. Dos Santos Silva RJ, Lima FA, Moura NM, Pardono E. Suicidal ideation and associated factors among adolescents in Northeastern Brazil. The Scientific World Journal. 2014; 2014:1-8.

\section{Notas}

* Artículo de investigación

Licencia Creative Commons CC BY 4.0

Como citar este artículo: Luna Contreras M, Dávila Cervantes CA. Adolescentes en riesgo: factores asociados con el intento de suicidio en México. RevGerencPolít Salud. 2017;17(34): 1-14. https://doi.org/10.11144/Javeriana.rgps17-34.arfa 